\title{
Efecto de sedimentos naturales enriquecidos con zinc, en modelos aislados y en microcosmos, sobre tres especies de invertebrados bentónicos
}

\author{
Marcela Galar Martínez, Laura Martínez-Tabche, Eugenia Sánchez-Hidalgo \& Eugenia López López \\ Laboratorio de Toxicología Acuática, sección de Graduados, Escuela Nacional de Ciencias Biológicas, Plan de Ayala \\ y Carpio s/n, Delegación Miguel Hidalgo, México, D.F. CP 11340, Fax (52)53966503; \\ marcela_galar_martinez@hotmail.com
}

Recibido 06-XII-2002. Corregido 17-IX-2003. Aceptado 12-XI-2003.

\begin{abstract}
Effect of zinc-enriched sediments, in open and isolated systems, on three species of benthonic invertebrates. Availability of toxics in aquatic bodies is limited by the physicochemical characteristics of sediments and water, as well as by the interactions between the different xenobiotics and inhabits species. The aim of this work was to relate the effect produced by zinc $(\mathrm{Zn})$ spiked in sediments of the Ignacio Ramírez dam (PIR), in isolated and microcosms models, on ATP concentration of three benthic organisms with the metal biodisponibility. The selected species were a crustacean, an annelid and a mollusk: Hyalella azteca (Amphipoda: Hyalellidae), Limnodrilus hoffmeisteri (Oligochaeta: Tubificidae) and Stagnicola attenuata (Basommatophora: Lymnaeidae), species that are found at high proportions in the reservoir and use different spaces in the benthos. Samples of sediments and organisms were collected from the PIR during the dry season (February of 1999). Metal concentration ( $\mathrm{Zn}, \mathrm{Fe}, \mathrm{Cu}$ and $\mathrm{Ni}$ ), $\mathrm{pH}$, texture, particle size, total nitrogen and organic matter were determined in sediments. Sublethal studies were carried out using two types of static systems (organisms isolated and in microcosms). Both models contained PIR sediments enriched with Zn (nominal concentration of 0.8129 $\mathrm{mg} / \mathrm{kg}$ ) and synthetic water in a proportion of 1:4. The test organisms were added to the systems once the equilibrium was reached $(2 \mathrm{hr})$ considering the biomass quantity with respect to volume $(1.0 \mathrm{~g}$ of organism by each $100 \mathrm{ml}$ of water:sediment). After 0, 12, 24, 36, 48 and $72 \mathrm{hr}$ of exposure, samples of sediment and hydrobionts were taken, and $\mathrm{Zn}$ content was quantified by atomic absorption. ATP concentration was also determined in organisms. The effect produced by natural sediments spiked with $\mathrm{Zn}$ is increased by the presence of more than one specie in the system (microcosm). With respect to $\mathrm{Zn}$ levels, two of the organisms tend to lose this metal in isolated and microcosm models, probably as a regulation strategy in its accumulation, as well as Fe presence in the reservoir sediments. Therefore, H. azteca maintained a constant $\mathrm{Zn}$ levels during the whole experiment. It is possible that the caption rate is so low in this organism that it does not need another regulatory mechanism. A decrement in ATP in worms and amphipods was observed in both systems at all exposure times. Possibly Zn and other metals present in the sediments interfere with the energy production enzymes by binding to SH groups. Nevertheless this biomolecule increased in snails in microcosms at all exposure times and in isolated systems at the end of the experiment, probably due to compensatory mechanisms and reduction in energy consumption present in mollusks during heavy metals exposure. Rev. Biol. Trop. 54(2): 451-460. Epub 2006 Jun 01.
\end{abstract}

Key words: ATP, Zn, sediments, Hyallela azteca, Limnodrilus hoffmeisteri, Stagnicola attenuata.

El modelo de microcosmos representa un buen acercamiento al estudio de los efectos de las sustancias tóxicas sobre ecosistemas acuáticos, ya que involucra comunidades pertenecientes a los cuerpos de agua a evaluar (Hendrix et al. 1981). La creación de estos sistemas se realiza generalmente con el agua, sedimento y organismos naturales de dicho ambiente, lo cual es útil porque de esta forma se incorporan diferentes elementos abióticos (tipo de sedimentos, entre otros), así como la interacción interespecies, factores que pueden 
modificar la biodisponibilidad de los xenobióticos en estudio y por lo tanto su toxicidad.

Así, la evaluación de la calidad de los sedimentos es determinante para la protección y manejo de los ecosistemas acuáticos (Smith et al. 1996). Ésta se encuentra influenciada por el ambiente y en especial por las especies que lo habitan, así como por sus características fisicoquímicas: $\mathrm{pH}$, salinidad, materia orgánica, etc. (Hakanson 1992). Para los organismos bentónicos estos parámetros son muy importantes, ya que determinan si es o no un habitat aceptable, especialmente en el caso de aquellos empleados en pruebas de toxicidad (Van del Hurk 1994). Un análisis fisicoquímico y toxicológico de este sustrato puede reflejar la calidad del sistema y la historia de la contaminación de una determinada área (El-Sammak y Aboul-Kassim 1999).

Uno de los aspectos más importantes del metabolismo es el control homeostático de ciertos iones inorgánicos y de la carga energética adenilada, ya que son factores interdependientes y esenciales para la supervivencia celular. En consecuencia se puede esperar que los contaminantes disminuyan la disponibilidad de ATP y perturben el transporte iónico (Repetto y Repetto 1995). La estimación de este parámetro en estudios de toxicidad de un xenobiótico puede proporcionar información sobre cambios en la fisiología de la célula y el alcance de su efecto.

Uno de los iones sometidos a dicho control homeostático es el zinc ( $\mathrm{Zn}$ ), ya que está implicado en un gran número de rutas fisiológicas. Sin embargo, la exposición a altas concentraciones de este metal resulta en efectos deletéreos para los diferentes organismos, ya que tiene alta afinidad por los grupos $\mathrm{SH}$ y $\mathrm{OH}$, así como gran capacidad complejante de aminoácidos, péptidos, proteínas y ácidos nucléicos, por lo que sus efectos pueden ser muy diversos. A concentraciones subletales ha demostrado ser desestabilizante de ADN, membranas, lisosomas y ribosomas, e interacciona con otros iones metálicos esenciales como $\mathrm{Ca}, \mathrm{Mg}, \mathrm{Cu}$ y Fe. Disminuye el contenido celular de los dos últimos, por lo tanto de ferritina, hemosiderina y ceruloplasmina, y altera a otras biomoléculas que los utilizan como cofactor (Moreno et al. 1999).

Estudios realizados por Bianchini y Carvalho (1999) demostraron el efecto inhibitorio del $\mathrm{Zn}$ sobre la $\mathrm{Na}^{+}, \mathrm{K}^{+}$-ATPasa de Chasmagnatus granulata y una reducción en la energía consumida por la actividad enzimática. Martínez-Tabche et al. (2000) mostraron que al ser sembrado en sedimentos de granjas trutícolas produce un decremento en las concentraciones de ATP, proteínas y hemoglobina del gusano Limnodrilus hoffmeisteri.

Por lo anterior, el objetivo de este estudio fue relacionar el efecto producido por los sedimentos del embalse Ignacio Ramírez (PIR) enriquecidos con $\mathrm{Zn}$, sobre la concentración de ATP de Stagnicola attenuata (Fay 1829), L. hoffmeisteri (Claparede 1862) y Hyalella azteca (Saussure 1857) con la captación del metal por los organismos, en modelos aislados $\mathrm{y}$ en microcosmos.

El Zn fue elegido como tóxico para este experimento debido a que este oligoelemento puede alcanzar concentraciones altas en los sedimentos, ya que en las inmediaciones de la PIR se llevan al cabo actividades de diversa índole (agrícolas, industriales y domésticas) que incorporan grandes cantidades de éste y otros contaminantes al embalse. Se trabajó con tres organismos bentónicos, dado que éstos se encuentran en gran abundancia en la PIR, y al ocupar espacios diferentes dentro del bentos, permiten una evaluación más completa del efecto de la matriz en estudio.

\section{MATERIALES Y MÉTODOS}

Área de estudio y muestreo. El presente estudio fue realizado en la PIR que se encuentra localizada en el rio La Gavia, a $22 \mathrm{~km}$ de la ciudad de Toluca (Estado de México) y a $5 \mathrm{~km}$ del rio Lerma $\left(19^{\circ} 27^{\prime} 35^{\prime \prime} \mathrm{N}\right.$ y $\left.99^{\circ} 46^{\prime} 25^{\prime \prime} \mathrm{W}\right)$. La macrofauna acuática incluye al menos tres especies endémicas: Cambarellus moctezumae, Chirostoma riojai y Girardinochtys multiradiatus. El embalse tiene una capacidad de 
almacenaje de $20.5 \times 10^{6} \mathrm{~m}^{3}$ y se emplea con fines agrícolas. El clima es templado con lluvias en el verano; los meses más cálidos son mayo y junio y la temperatura ambiental media anual es de $12.4^{\circ} \mathrm{C}$. La deforestación, prácticas agrícolas ineficientes y suelos con alta pendiente son problemas comunes en esta área y contribuyen notablemente al deterioro ambiental de la zona (De la Vega et al. 1997).

El muestreo fue realizado en la compuerta, en febrero de 1999 (estación de sequía). Las muestras de sedimento se tomaron utilizando una draga cónica de acero inoxidable; en el mismo sitio se colectaron las tres especies de prueba: gusanos (L. hoffmeisteri), anfípodos (H. azteca) y caracoles (S. attenuata).

Cultivo y mantenimiento de los organismos. Los organismos colectados en la PIR fueron separados y trasladados al laboratorio. Posteriormente se colocaron en acuarios con arena (tamaño de partícula de $0.84 \mathrm{~mm}$ ) y agua sintética $\left(0.22 \mathrm{~g}\right.$ de $\mathrm{MgSO}_{4}, 0.18 \mathrm{~g}$ de $\mathrm{NaHCO}_{3}$, $0.08 \mathrm{~g}$ de $\mathrm{KCl}$ y $0.13 \mathrm{~g}$ de $\mathrm{CaSO}_{4} \cdot 2 \mathrm{H}_{2} \mathrm{O}$ por litro) en una proporción de 1:4; fueron mantenidos a temperatura ambiente, ciclos de luz: oscuridad naturales y oxigenación constante (6.4-6.6 mg/l) durante 7 días antes de iniciar el experimento. La alimentación en esta fase fue ad libitum: los gusanos con melaza al $5 \%$ diluida en cloruro de sodio $(0.9 \%)$; los anfípodos y caracoles con lechuga. Durante el tratamiento a los organismos no se les proporcionó alimento; sin embargo, los tres tienen la capacidad de consumir el detritus presente en los sedimentos de la PIR.

Caracterización de los sedimentos de la PIR. Los sedimentos colectados fueron secados en una estufa a $80^{\circ} \mathrm{C}$ y tamizados a través de malla de acero inoxidable de tamaño 0.02 $\mathrm{mm}$. A este polvo se le determinó: $\mathrm{pH}$, materia orgánica, textura, nitrógeno total y la concentración de $\mathrm{Zn}, \mathrm{Ni}, \mathrm{Fe}$ y $\mathrm{Cu}$, por espectroscopía de absorción atómica (Anónimo 1995).

Determinación del tiempo al equilibrio del $\mathrm{Zn}$ en los sedimentos de la PIR. Se formaron diez lotes de $10 \mathrm{~g}$ de sedimento. Estas muestras se mezclaron con $0.2 \mathrm{mg} / \mathrm{kg}$ de $\mathrm{Zn}$ $\left(\mathrm{ZnSO}_{4}\right.$, Sigma) y agua sintética en proporción 1:4. Posteriormente se agitaron mecánicamente en vasos de plástico, alcanzando una concentración nominal de $0.8129 \mathrm{mg} / \mathrm{kg}$ (Zn en sedimentos más el adicionado). Después de 1, 2, 3, 4 , y $5 \mathrm{hr}$, las muestras se centrifugaron a 3000 $\mathrm{x}$ g por 15 min y la concentración del metal fue cuantificada en el agua y sedimentos por absorción atómica (Varian modelo AA-1475). Este metal rápidamente alcanzó el equilibrio con el material partículado $(2 \mathrm{hr})$.

Estudio de toxicidad subletal de $\mathrm{Zn}$ en sedimentos de la PIR. Conociendo el tiempo de equilibrio, se prepararon los sistemas de intoxicación: sedimentos de la PIR enriquecidos con $0.2 \mathrm{mg} / \mathrm{kg}$ de $\mathrm{Zn}$ y agua sintética (1:4). La concentración de $0.8129 \mathrm{mg} / \mathrm{kg}$ del metal se encuentra dentro del intervalo de la $\mathrm{CL}_{0}$ de $H$. azteca, organismo que resultó ser el más sensible a este sistema, comparado con L. hoffmeisteri y S. attenuata (M. Galar y L. Martínez-Tabche, en prep.). Posteriormente se colocaron los organismos de prueba (caracol, anfípodo y gusano), en forma aislada y en microcosmos (las tres especies juntas), considerándose la cantidad de biomasa con respecto a volumen ( $1 \mathrm{~g}$ de organismo por cada $100 \mathrm{ml}$ de sistema sedimento:agua). Los sistemas de intoxicación fueron estáticos. Después de 0, $12,24,36,48$ y $72 \mathrm{hr}$ de exposición, se tomaron muestras de organismo y sedimento para cuantificar la concentración de Zn; a los organismos también se les determinaron los niveles de ATP. En los grupos testigo el sedimento de la PIR fue sustituido con arena. Se efectuaron cinco réplicas de cada uno de los sistemas y tiempos de exposición.

Determinación de ATP. De cada una de las tres especies de prueba, tanto aislados como en microcosmos, se pesaron $0.5 \mathrm{~g}$ de tejido (los caracoles fueron desprovistos de la concha y solo se utilizó el tejido blando); éste se cortó finamente y se le adicionaron $20 \mathrm{ml}$ de buffer de fosfatos a $\mathrm{pH}$ 7. Posteriormente 
se homogeneizó y centrifugó (ultracentrífuga Hermle) durante $15 \mathrm{~min}$ a $12500 \mathrm{rpm} \mathrm{a}-5^{\circ} \mathrm{C}$. Finalmente se separó el sobrenadante, el cual se utilizó para la determinación de la concentración de ATP por el método de Adams (1963), el cual se fundamenta en la reacción entre el 3- fosfoglicerato y el ATP catalizada por la fosfoglicerato cinasa y la fosfato gliceraldehído deshidrogenasa. Esta reacción involucra la oxidación de NADH; el cambio en la absorbancia permite la cuantificación de NAD.

Cuantificación de Zn. Se tomaron $0.5 \mathrm{~g}$ de la muestra (sedimento u organismos), se adicionaron $5 \mathrm{ml}$ de ácido nítrico concentrado y se realizó una digestión en autoclave por $1 \mathrm{hr}$ a $121^{\circ} \mathrm{C}$ y $15 \mathrm{lbs}$ de presión. Posteriormente las muestras se diluyeron con agua desionizada hasta alcanzar un volumen de $25 \mathrm{ml}$ y se evaluó la cantidad de $\mathrm{Zn}$ mediante espectroscopía de absorción atómica.

Análisis Estadístico. Los datos experimentales fueron sometidos a un análisis de varianza bifactorial (ANOVA) y las diferencias de las medias de cada grupo fueron comparadas contra el tiempo cero (ya que no se encontró diferencia significativa entre éste y los testigos), utilizando el método de Duncan. Éstas fueron consideradas significativas a $\mathrm{p}<0.05$.

\section{RESULTADOS}

Los resultados del estudio sobre las características fisicoquímicas de los sedimentos de la PIR muestran que éstos tienen un $\mathrm{pH}$ de 6.5 en promedio. Se encuentran constituidos en su mayoría por limo (81.8\%), siendo los porcentajes de arena (13.45\%) y arcilla (4.65\%) mucho menores. Por lo que respecta al tamaño de partícula, ésta tiene una dimensión aproximada de $177 \mu$. La cantidad de nitrógeno total encontrado fue de $1.74 \mathrm{mg} / \mathrm{kg}$ y la de materia orgánica de $4.9 \mathrm{mg} / \mathrm{kg}$.

En cuanto a los metales residuales contenidos en los sedimentos de la PIR, se observó que contienen una gran proporción de $\mathrm{Fe}$
$(30.3130 \mathrm{mg} / \mathrm{kg})$, y que también se encuentran presentes $\mathrm{Zn}$, Ni y $\mathrm{Cu}$, aunque en menor concentración $(0.6129,0.3360$ y $0.1070 \mathrm{mg} / \mathrm{kg}$, respectivamente).

El estudio del tiempo al equilibrio demostró que el sedimento de la PIR alcanza concentraciones similares entre el agua y la matriz en $2 \mathrm{hr}$.

En el estudio subletal los caracoles y gusanos expuestos en forma aislada perdieron $\mathrm{Zn}$ endógeno, alcanzando una disminución de 49 y $59 \%$ respectivamente $(\mathrm{p}<0.05)$, a las $72 \mathrm{hr}$ de exposición (Figs. 1 y 3). En contraste, en el anfípodo aún cuando la concentración del metal tiende a incrementarse hasta un $14.72 \%$ $(72 \mathrm{hr}$ ), el efecto no fue significativo (Fig. 5). En el modelo de microcosmos se observó el mismo comportamiento, ya que la concentración del metal disminuyó en el caracol y gusano $43.45 \%$ y $28.22 \%$ respectivamente $(\mathrm{p}<0.05)$, mientras que en $H$. azteca no hubo variación significativa (Figs. 2, 4 y 6).

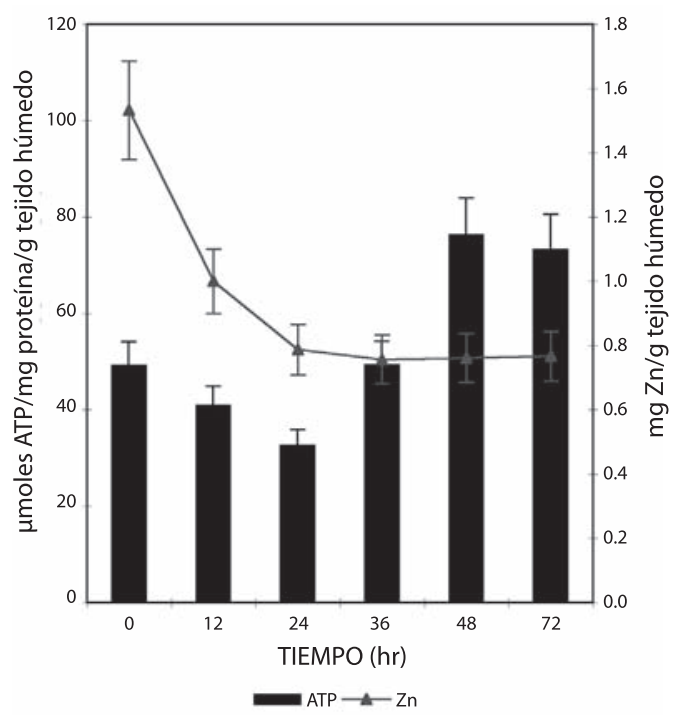

Fig. 1. Efecto de sedimentos de la PIR enriquecidos con $\mathrm{Zn}$ sobre la concentración de ATP y Zn de Stagnicola attenuata en sistemas aislados.

Fig. 1. Effect of PIR sediments enriched with $\mathrm{Zn}$ on ATP and $\mathrm{Zn}$ concentration of Stagnicola attenuata in isolated systems. 


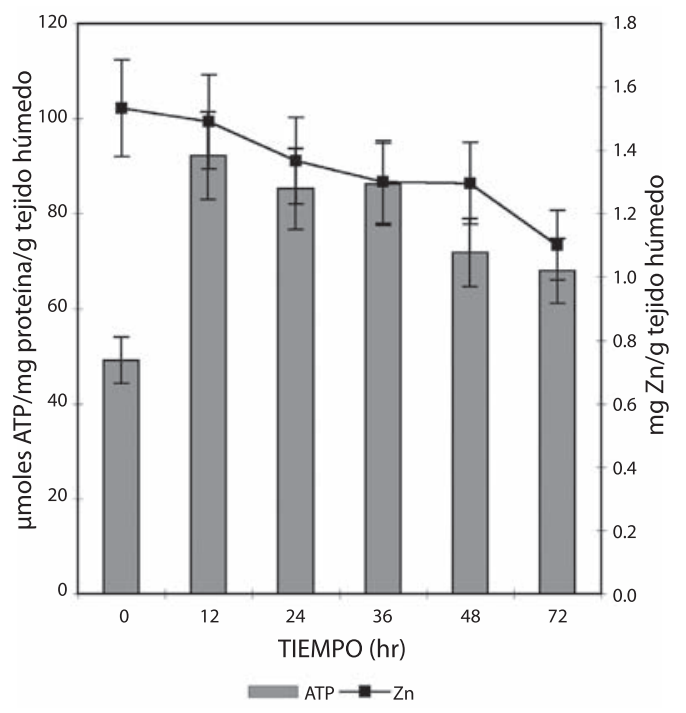

Fig. 2. Efecto de sedimentos de la PIR enriquecidos con $\mathrm{Zn}$ sobre la concentración de ATP y Zn de Stagnicola attenuata en sistemas en microcosmos.

Fig. 2. Effect of PIR sediments enriched with Zn on ATP and $\mathrm{Zn}$ concentration of Stagnicola attenuata in microcosms systems.

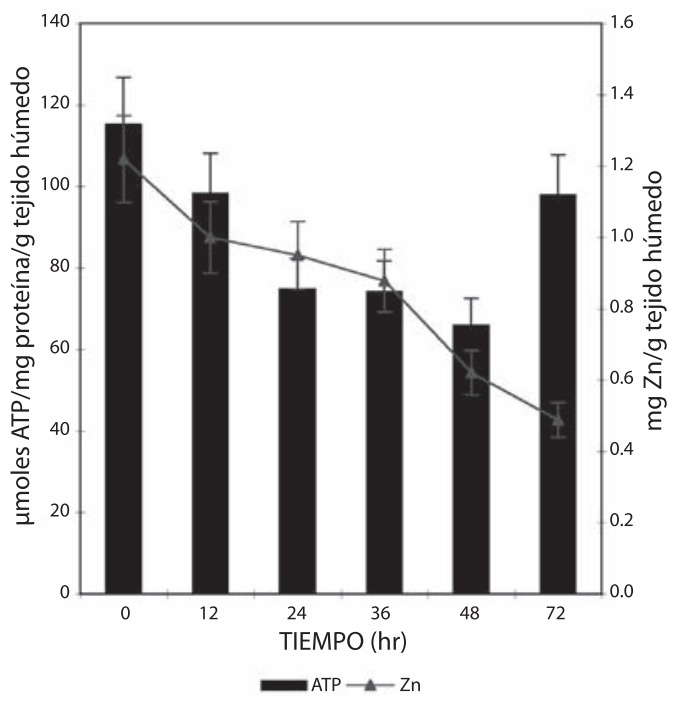

Fig. 3. Efecto de sedimentos de la PIR enriquecidos con Zn sobre la concentración de ATP y Zn de Limnodrilus hoffmeisteri en sistemas aislados.

Fig. 3. Effect of PIR sediments enriched with Zn on ATP and $\mathrm{Zn}$ concentration of Limnodrilushoffmeisteri in isolated systems.
En los gusanos y anfípodos expuestos en forma aislada, la concentración de ATP disminuyó (Figs. 3 y 5). En el primer organismo, el efecto máximo se observó a las 48 horas $(\mathrm{p}<0.05)$; sin embargo, este efecto fue más significativo en el último, ya que el decremento que alcanzó fue de $65.4 \%$ a las $72 \mathrm{hr}$ de exposición ( $\mathrm{p}<0.05$ ). En este sistema la reducción de ATP también se observó en el caracol, pero únicamente a las 12 y $24 \mathrm{hr}$ de intoxicación, ya que a los tiempos de 48 y $72 \mathrm{hr}$, éste se incrementó en 55 y $48.9 \%$ respectivamente $(\mathrm{p}<0.05)$ (Fig. 1).

Cuando los organismos se expusieron en microcosmos, se observó un efecto similar al encontrado en sistemas aislados, esto es, un decremento en la concentración de ATP tanto en gusanos como en anfípodos. En L. hoffmeisteri la concentración mínima de ATP se alcanzó a las $24 \mathrm{hr}$, obteniéndose una reducción del 79\% $(\mathrm{p}<0.03)$ (Fig. 4); en $H$. azteca, el máximo decremento se encontró a las $72 \mathrm{hr}$, siendo de $89 \%(\mathrm{p}<0.05)$ (Fig. 6). Sin embargo, en el caracol se encontró un

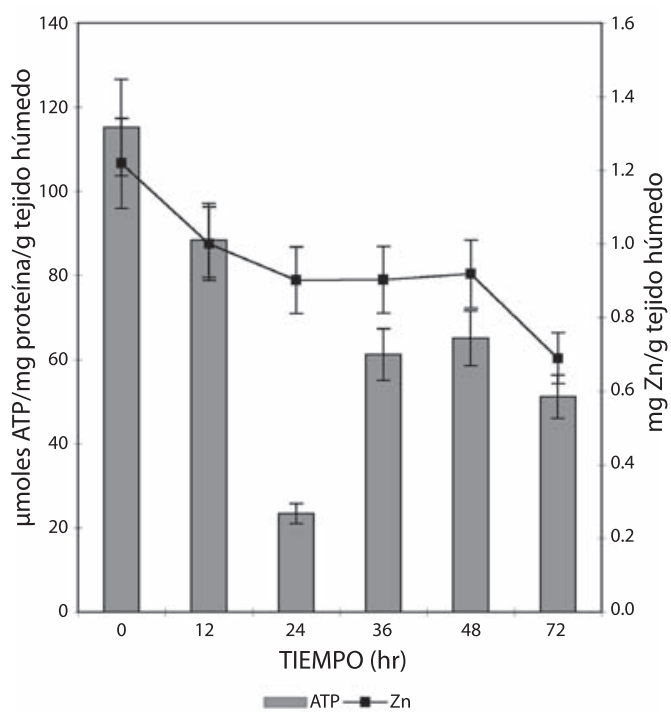

Fig. 4. Efecto de sedimentos de la PIR enriquecidos con Zn sobre la concentración de ATP y Zn de Limnodrilus hoffmeisteri en sistemas en microcosmos.

Fig.4. Effect of PIR sediments enriched with Zn on ATP and $\mathrm{Zn}$ concentration of Limnodrilus hoffmeisteri in microcosms systems. 


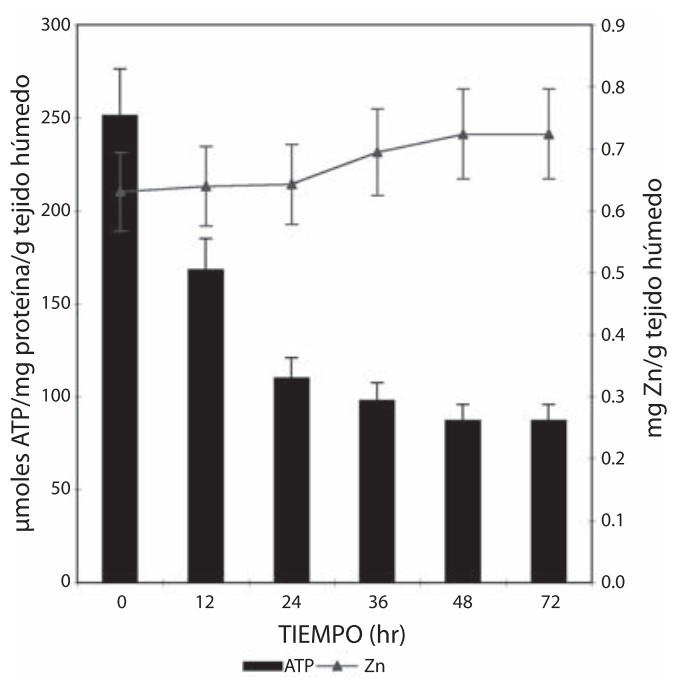

Fig. 5. Efecto de sedimentos de la PIR enriquecidos con $\mathrm{Zn}$ sobre la concentración de ATP y Zn de Hyalella azteca en sistemas aislados.

Fig. 5. Effect of PIR sediments enriched with $\mathrm{Zn}$ on ATP and Zn concentration of Hyalella azteca in isolated systems.

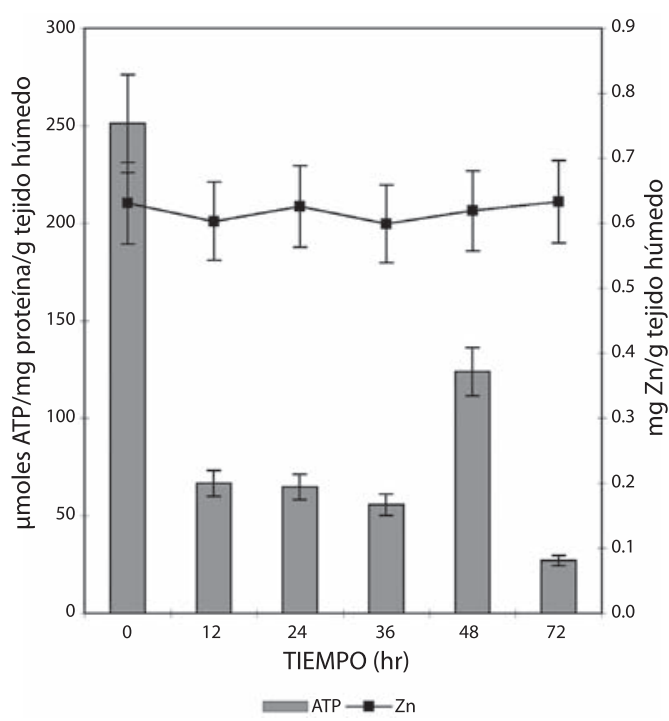

Fig. 6. Efecto de sedimentos de la PIR enriquecidos con $\mathrm{Zn}$ sobre la concentración de ATP y Zn de Hyalella azteca en sistemas en microcosmos.

Fig. 6. Effect of PIR sediments enriched with Zn on ATP and $\mathrm{Zn}$ concentration of Hyalella azteca in microcosms systems. incremento de ATP a todos los tiempos de exposición: 87.4, 73.2, 75.1, 45.8 y 38.1\%, respectivamente $(\mathrm{p}<0.05)$ (Fig. 2).

\section{DISCUSIÓN}

Los sedimentos de la PIR están constituidos principalmente por limo y materia orgánica. Calmano (1996) menciona el papel relevante de esta última y de la relación limo/arcilla para enlazar metales, y por consiguiente, para su transferencia dentro de los sistemas biológicos. De acuerdo a esto, cabe suponer que la composición de los sedimentos de la PIR le permiten una alta disponibilidad al $\mathrm{Fe}, \mathrm{Zn}$, Ni y $\mathrm{Cu}$ presentes en este cuerpo de agua; ésta contribuirá a que la captación de dichos metales, y por consiguiente su toxicidad sobre los tres organismos expuestos a este sustrato, pueda ser alta. Giesy y Hoke (1990) señalan que los sedimentos no son peligrosos para los organismos acuáticos cuando estos elementos se encuentran por debajo de $17000,90,20$, y $25 \mathrm{mg} / \mathrm{kg}$ respectivamente, por lo que con las concentraciones encontradas en el presente estudio (30.3130, $0.6129,0.3360$ y $0.1070 \mathrm{mg} / \mathrm{kg}$ ), la zona muestreada no sería peligrosa para la vida acuática. Sin embargo, los resultados obtenidos sobre los tres organismos, tanto en sistemas aislados como en microcosmos, demuestran que la exposición a los sedimentos de la PIR enriquecidos con $\mathrm{Zn}$ produce cambios notables en las concentraciones de ATP. Es importante considerar que la presencia de otros contaminantes en este sustrato natural puede modificar la biodisponibilidad y respuesta tóxica inherente a cada uno de los xenobióticos aislados. Al respecto, Favari et al. (2002) encontraron que los sedimentos de la PIR contienen insecticidas organofosforados; estos compuestos producen cambios en el metabolismo de la glucosa, y por lo tanto pueden influir en la regulación de ATP (Heat 1987).

En este estudio se observó que en el anfípodo y el gusano la concentración de ATP disminuyó a todos los tiempos de exposición, tanto en sistemas aislados como en microcosmos. 
Estos resultados concuerdan con los obtenidos por Martínez-Tabche et al. (2000), quienes al exponer al gusano L. hoffmeisteri a diferentes concentraciones de $\mathrm{Zn}$, encontraron un decremento en el nivel de ATP. Así mismo, Sanders et al. (1996) aseveran que los metales disminuyen el suministro de energía. Tort et al. (1982) consideran que este daño se debe a la inhibición de las enzimas mitocondriales responsables del proceso de respiración. Otra posible explicación a la disminución de ATP sería que el Fe contenido en los sedimentos de la PIR puede bioconcentrarse y alterar la producción de esta biomolécula, ya que el exceso de este metal activa la reducción férrica en la membrana mitocondrial interna, lo que genera que se interrumpa inmediatamente la cadena de los citocromos y por ende la producción de ATP (Repetto y Repetto 1995).

En los caracoles expuestos en forma aislada hasta las $24 \mathrm{hr}$ de exposición, también se observó un decremento en la concentración de ATP. Sin embargo, a partir de las $36 \mathrm{hr}$ $\mathrm{y}$ en el modelo en microcosmos a todos los tiempos de exposición, esta biomolécula se vio incrementada, resultando diferente el comportamiento de este organismo con respecto al de las otras dos especies en estudio. Existen diversos trabajos que demuestran que la respuesta tóxica producida por los metales en los hidrobiontes depende de su concentración y de la especie. Biachini et al. (1999) observaron una alta tolerancia del crustáceo $C$. granulata hacia Zn. Spear (1981) encontró en organismos de la misma clase de peces, que el orden de los Perciformes es el grupo más resistente y el Clupeiforme el más sensible hacia la exposición a Zn. Para explicar el aumento de ATP en los caracoles, también es necesario considerar lo encontrado por Spicer y Weber (1991), quienes mencionan que en general el consumo de oxígeno en moluscos es reducido cuando se encuentran expuestos a metales pesados y por lo tanto la utilización de energía se ve disminuida, acumulando altos niveles de ATP. Otra posible explicación sería que aún cuando el $\mathrm{Zn}$ tiene una gran capacidad complejante con grupos SH y SS de las enzimas de la cadena respiratoria, es probable que los moluscos expuestos a este metal (en ambos modelos) hayan desarrollado mecanismos compensatorios hacia el estrés producido por la exposición al sistema.

Algunos invertebrados marinos son capaces de acoplar las tasas de excreción de metales con las de captación, de tal manera que regulan la concentración corporal (Rainbow 1990). En este estudio el caracol y el gusano expuestos tanto en forma aislada como en microcosmos, perdieron $\mathrm{Zn}$, probablemente porque su sistema de regulación esté en la fase inicial. Un efecto similar fue encontrado por Nugegoda y Rainbow (1989) en camarones expuestos al mismo; éstos regulan las concentraciones corporales de este metal durante cortos periodos de tiempo incrementando su excreción, aun cuando se encuentre altamente biodisponible. Aunque la regulación de metales no es una estrategia de acumulación generalizada en los invertebrados, se encuentran algunos ejemplos de moluscos como Haliotis tuberculata (Bryan et al. 1977) y poliquetos como Nereis diversicolor (Bryan y Hummerstone 1973), que utilizan mecanismos similares para la regulación de las concentraciones corporales de $\mathrm{Zn}$. Es posible entonces, que tanto $S$. attenuata como L. hoffmeisteri posean mecanismos de regulación semejantes.

Otra posible explicación a la pérdida de $\mathrm{Zn}$ en gusanos y caracoles es la presencia de Fe en los sedimentos de la PIR. Prusod y Beck (1996) demostraron que este metal en altas concentraciones disminuye la entrada de $\mathrm{Zn}$ en diferentes organismos, favoreciéndose la captación de Fe. Es probable entonces que las especies en estudio estén acumulando este último, y como consecuencia se induzca la producción de metalotioneínas, formando complejos tanto con éste como con otros metales, entre ellos el $\mathrm{Zn}$ endógeno. Estos quelatos son susceptibles de oxidación, y cuando se encuentran presentes en grandes cantidades se polimerizan y acumulan en lisosomas, y pueden posteriormente ser expulsados del organismo por exocitosis (Rainbow 1990).

En este estudio la pérdida del metal fue mayor cuando ambos organismos se 
encontraban en sistemas aislados. Estudios realizados por Ciarelli et al. (2000) demostraron que la captación de fluoroetano en el poliqueto Nereis viren fue menor al introducir el anfípodo Corophium volutato.

Las concentraciones de $\mathrm{Zn}$ en $H$. azteca, en ambos sistemas, se mantienen constantes. Rainbow (1990) menciona que algunos invertebrados marinos pueden disminuir la captación de metales por lo que no requieren estrategias de regulación, tal es el caso del anfípodo Echinoggamarus pirloti, que muestra pocos cambios en la acumulación neta de Zn corporal aun cuando se incremente la biodisponibilidad del mismo (Rainbow y White 1989).

Los resultados obtenidos en este estudio muestran que la respuesta tóxica se ve modificada por la presencia de más de una especie en el sistema (microcosmos), ya que fue más evidente el efecto del Zn sobre las concentraciones de ATP cuando se expusieron los tres organismos juntos. Es importante considerar que si bien las tres especies en estudio viven en el sedimento, su permanencia en el mismo y la exposición a los xenobióticos presentes es diferente. El gusano es un organismo bentónico estricto, que permanece en esta matriz durante toda su vida, mientras que el caracol y el anfípodo son bentónicos facultativos, por lo que se desplazan también en la columna de agua y el contacto con el sedimento y sus contaminantes es menor. Así mismo, es probable que los hidrobiontes estén removiendo otros xenobióticos presentes en el sustrato, incrementando la biodisponibilidad de los metales y otros tóxicos, y por lo tanto potenciando su efecto. Adicionalmente, no se puede descartar que las diferentes especies liberen metabolitos tóxicos que tengan efecto sobre las otras, o que modifiquen las condiciones ambientales del sistema como el oxígeno disuelto, $\mathrm{pH}$ y nitrógeno total, entre otros. Rico Martínez et al. (1998) observaron reducción en la densidad de población y diversidad de especies de rotíferos al exponerlos a $\mathrm{Cu}$ en microcosmos, posiblemente debido a cambios ambientales ocasionados por las mismas especies en el sistema. Otro trabajo que apoya esta hipótesis es el estudio realizado por Sibley et al. (2001) con cladóceros, rotíferos y copépodos expuestos a creosota en microcosmos, donde se observaron cambios en el tipo y proporción de especies de invertebrados, probablemente por alteración del $\mathrm{pH}$ en el medio y en consecuencia cambios en la biodisponibilidad del compuesto.

En conclusión, este trabajo demuestra que la respuesta generada por los sedimentos de la PIR enriquecidos con $\mathrm{Zn}$ es más tóxica cuando los organismos se encuentran en microcosmos, por lo que es necesaria la utilización de sistemas multiespecies para la evaluación de la contaminación acuática. Así mismo, manifiesta la importancia de considerar a los sedimentos en pruebas de toxicidad, ya que los contaminantes presentes en este sustrato pueden producir efectos adicionales.

\section{RESUMEN}

En los cuerpos acuáticos la disponibilidad de los tóxicos es limitada por las características fisicoquímicas de los sedimentos y agua, así como por las interacciones entre los diversos xenobióticos y entre las especies que los habitan. Por lo anterior, el objetivo del presente trabajo fue relacionar el efecto producido por el zinc (Zn) sembrado en sedimentos del embalse Ignacio Ramírez (PIR), en modelos aislados y en microcosmos, sobre el ATP de tres organismos bentónicos con la biodisponibilidad del metal. Las especies seleccionadas fueron: Hyalella azteca, Limnodrilus hoffmeisteri y Stagnicola attenuata, dado que se encuentran en altas proporciones en el embalse y ocupan espacios diferentes en el bentos. Las muestras de sedimentos y organismos se colectaron en la estación de sequía (febrero de 1999). A los primeros se les determinó el $\mathrm{pH}$, textura, tamaño de partícula, nitrógeno total, materia orgánica y concentración de metales $(\mathrm{Zn}, \mathrm{Fe}, \mathrm{Cu}$ y Ni). Posteriormente se realizó un estudio de toxicidad subletal utilizando dos tipos de sistemas estáticos (organismos aislados y en microcosmos). Ambos modelos contenían sedimentos de la PIR enriquecidos con Zn (concentración nominal de $0.8129 \mathrm{mg} / \mathrm{kg}$ ) y agua sintética en una proporción de 1:4. Los organismos de prueba fueron adicionados una vez alcanzado el equilibrio $(2 \mathrm{hr})$ considerando la cantidad de biomasa con respecto al volumen (1.0 g de organismo por cada $100 \mathrm{ml}$ de agua:sedimento). Después de $0,12,24,36,48$ y $72 \mathrm{hr}$ de exposición se tomaron muestras del sedimento y de los hidrobiontes, a los cuales se les cuantificó el contenido de $\mathrm{Zn}$ por absorción atómica. A los organismos también se les determinó la concentración de ATP. Los resultados obtenidos muestran que el efecto 
producido por los sedimentos naturales enriquecidos se ve potenciado por la presencia de más de una especie en el sistema (microcosmos). Con respecto a los niveles de $\mathrm{Zn}$, dos de los organismos (L. hoffmeisteri y S. attenuata) tienden a perder este metal tanto en sistemas aislados como en microcosmos, probablemente como estrategia de regulación en la acumulación del mismo, así como debido a la presencia de $\mathrm{Fe}$ en los sedimentos del embalse. Sin embargo, H. azteca mantiene sus niveles de $\mathrm{Zn}$ constantes durante todo el experimento, ya que es posible que la tasa de captación sea $\tan$ baja que no requiera otro tipo de mecanismo de regulación. Por otra parte se observó una disminución de ATP tanto en gusanos como en anfípodos expuestos a ambos sistemas a todos los tiempos de exposición; posiblemente los metales que se encuentran en los sedimentos interfieren con las enzimas encargadas de la producción energética por unión a grupos $\mathrm{SH}$. Esta biomolécula se incrementó en el caracol en microcosmos a todos los tiempos de exposición y en sistemas aislados al final del experimento, probablemente debido a mecanismos compensatorios y al consumo reducido de energía que muestran los moluscos frente al estrés generado por la presencia de metales pesados.

Palabras clave: ATP, Zn, sedimentos, Hyalella azteca, Limnodrilus hoffmeisteri, Stagnicola attenuata.

\section{REFERENCIAS}

Adams, H. 1963. Adenosine-5'-triphosphate, determination with phosphoglycerate kinase, p. 539-543. In H. Urlich Bergmeyer (ed.). Methods of enzymatic analysis. Academic, Nueva York, EEUU.

Anónimo. 1995. Standard methods for the examination of water and wastewater. D.A. Eaton, L.S. Clesceri \& A.E. Greenberg (eds.). APHA, AWWA \& WPCF. Washington, D.C. 81-90 p.

Biachini, A. \& P. Carvalho de Castillo. 1999. Effects of zinc exposure on oxygen consumption and gill $\mathrm{Na}^{+}$, $\mathrm{K}^{+}$-ATPase of the estuarine crab Chasmagnathus granulata Dana, 1891 (Decapoda-Grapsidae). Bull. Environ. Contam. Toxicol. 62: 63-69.

Bryan, G.W. \& L.G. Hummerstone. 1973. Adaptation of the polychaete Nereis diversicolor to estuarine sediments containing high concentrations of zinc and cadmium, p. 71. In W.R. Furness \& P.S. Rainbow (eds.). Heavy metals in the marine environment. CRC, Boca Ratón, Florida, EEUU.

Bryan, G.W., G.W. Potts, \& G.R. Forster. 1977. Heavy metals in the gastropod mollusk Haliotis tuberculat, p. 71. In W.R. Furness \& P.S. Rainbow (eds.). Heavy metals in the marine environment. CRC, Boca Ratón, Florida, EEUU.
Calmano, W. 1996. Metals sediments: Removilization and environmental hazards, p. 1-14. In M. Munawar \& G. Dave (eds.). Development and progress in sediment quality assessment: Rationale, challenges, techniques and strategies. SPB, Amsterdam, Holanda.

Ciarelli, B.J., N.M. Kater, P. Van Straalen. 2000. Influence of bioturbation by the amphipod Corophium volutator on fluoranthene uptake in the marine polychaete Nereis virens. Environ. Toxicol. Chem. 19: 15751581.

De la Vega, S.M., L. Martínez-Tabche \& L.G. Macías. 1997. Bioaccumulation of methyl-parathion and toxicity in several species of fresh water community in Ignacio Ramírez dam in México. Ecotoxicol. Environ. Safe. 38: 53-62.

El-Sammak, A.A. \& T.A. Aboul-Kassim. 1999. Metal pollution in the sediments of Alexandria region, Southeastern Mediterranean, Egypt. Bull. Environ. Contam. Toxicol. 63: 263-270.

Favari, L., E. López, L. Martínez- Tabche \& E. Díaz-Pardo. 2002. Effect of insecticides on plankton and fish of Ignacio Ramírez reservoir (México): A biochemical and biomagnification study. Ecotox. Environ. Safe. 51: 177-186.

Giesy, J.P. \& R.A. Hoke. 1990. Freshwater sediment quality criteria: Toxicity bioassessment, p. 190. In R. Baudo, J. Giesy \& H. Muntau (eds.). Sediments: Chemistry and toxicity of in-place pollutants. Lewis, Chelsea, Michigan, EEUU.

Hakanson, L. 1992. Sediment variability, p. 19-34. In G.A. Burton (ed.). Sediment toxicity assessment. SETAC, Boca Ratón, Florida, EEUU.

Heat, A.G. 1987. Water pollution and fish physiology. CRC, Boca Ratón, Florida, EEUU 173-174 p.

Hendrix, P.F., C.L. Langer, E.P. Odum \& C.L. Thomas. 1981. Microcosms as test systems for ecotoxicological effects of toxic substances: An appraisal with cadmium. U.S. Environ. Protection Agency. EPA600/s3-81-036

Martínez-Tabche, L., C.I. Gutiérrez, O.L. Gómez, M.M. Galar \& F.C. Germán. 2000. Toxic effects of zinc from trout farm sediments on ATP, protein and haemoglobin concentrations on Limnodrilus hoffmeisteri. Toxicol. Environ. Health 59: 575-583.

Moreno, S. R., F. Díaz-Barriga \& S. Devars. 1999. Mecanismos de toxicidad y de tolerancia a los metales pesados, p. 12 -20. In C. Cervantes \& S.R. Moreno (eds.). Contaminación ambiental por metales pesados. AGT, México, D.F. 
Nugegoda, D. \& P.S. Rainbow. 1989. Effects of salinity changes on zinc uptake and regulation by the decapod crustaceans Palaemon elegans and Palaemonetes varians, p. 71. In W.R. Furness \& P.S. Rainbow (eds.). Heavy metals in the marine environment. CRC, Boca Ratón, Florida, EEUU.

Prusod, A.S. \& F.W.J. Beck. 1996. Metal-metal interactions, p. 189-197. In W. Chang (ed.). Toxicology of metals. Lewis, Boca Ratón, Florida, EEUU.

Rainbow, P.S. \& S.L. White. 1989. Comparative strategies of heavy metal accumulation by crustaceans: Zinc, copper and cadmium in a decapod, an amphipod and a barnacle, p. 72. In W.R. Furness \& P.S. Rainbow (eds.). Heavy metals in the marine environment. CRC, Boca Ratón, Florida, EEUU.

Rainbow, P.S. 1990. Heavy metal levels in marine invertebrates, p. 68-73. In W.R. Furness \& P.S. Rainbow (eds.). Heavy metals in the marine environment CRC, Boca Ratón, Florida, EEUU.

Repetto, G. \& M. Repetto. 1995. Métodos alternativos: estudios toxicológicos in vitro, p. 37-59. In $\mathrm{M}$. Repetto (ed.).Toxicología avanzada. Díaz de Santos, Madrid.

Rico-Martínez, R., I. Pérez-Lagaspi, G.E. QuinteroDíaz, M.G. Rodríguez-Martínez, M.A. HernándezRodríguez \& J.E. Zaragoza-Almaraz. 1998. Effects of copper addition to laboratory maintained microcosms of Presidente Calles reservoir organisms (Aguascalientes, México). Aqua. Ecosys. Health Manag. 1: 323-332.
Sanders M.B., L.P. Goering \& K. Jenkins. 1996. The role of general and metal-specific cellular responses in protection and repair of metal-induced damage: Stress proteins and metallothioneines, p. 165. In W. Chang (ed.). Toxicology of metals. Lewis, Boca Ratón, Florida, EEUU.

Sibley, M.L., K.T. Harris, T.A. Bestari, R.D. Steele, R.W. Robinson, K.E. Gensemer, K.R. Day \& R.S. Solomon. 2001. Response of zooplankton communities to liquid creosote in freshwater microcosms. Environ. Toxicol. Chem. 2: 394-405.

Smith, L.S., D.D. MacDonald, K.A. Keenleyside, C.D. Ingersoll \& L.J. Field. 1996. A preliminary evaluation of sediment quality assessment values for freshwater ecosystems. K. Great Lakes Res. 22(3): 621-638.

Spear, P.A. 1981. Zinc in the aquatic environment: Chemistry, distribution and toxicology, p. 70-74. In E.W. Taylor (ed.). Toxicology of aquatic pollution, physiological, cellular and molecular approaches. Cambridge University, Cambridge, Inglaterra.

Spicer, J.I. \& R.E. Weber. 1991. Respiratory impairment in crustaceans and mollusks due to exposure to heavy metals. Comp. Biochem. Physiol. 100C: 339-342.

Tort, L., S. Crespo \& J. Balasch. 1982. Oxygen consumption of the dogfish gill tissue following zinc treatment. Comp. Biochem. Physiol. 72C: 145-151.

Van del Hurk, P. 1994. Effects of natural sediment properties on test results in bioassays with oyster larvae (Crassostres gigas) on sediment elutriates. J. Aquat. Ecosys. Health 3: 185-191. 